\title{
Influencia del género en el desarrollo del trastorno psicosomático: revisión narrativa
}

\author{
Influence of gender on the development of psychosomatic disorder: a narrative review \\ Influência do gênero no desenvolvimento do transtorno psicossomático: revisão narrativa
}

\author{
Elisabet Torrubia-Pérez ${ }^{\circledR}$; Silvia Reverté-Villarroya ${ }^{\prime \prime \prime l} @$; Maria-Antonia Martorell-Povedal @
}

'Universitat Rovira i Virgili. Tarragona, Espanha; "Instituto de Investigación Sanitaria Pere Virgili, Espanha

\begin{abstract}
RESUMEN
Objetivo: describir los factores psicosociales y culturales que puedan determinar el proceso psicosomático, analizar la influencia que el género tiene sobre su desarrollo y reflexionar sobre su abordaje. Método: se realiza una revisión narrativa, reuniendo contenido fundamentado sobre psicosomatización y malestares sin causa orgánica aparente que tuvieran en cuenta el género o aporten fundamentación teórica. Se consultan las bases de datos Dialnet, Scielo, Google Académico y CUIDEN, así como libros, documentos y análisis estadísticos de interés. Resultados: se seleccionan un total de diez artículos disponibles en red, cinco artículos en formato papel, ocho libros y monográficos y tres informes estadísticos. Conclusión: la literatura refleja que las desigualdades de género pueden condicionar el desarrollo de trastornos psicosomáticos en relación a los factores psicosociales. Ello se traduce en una notoria morbilidad diferencial. Ante ello, es necesario destacar la importancia de la atención sanitaria holística, desde un enfoque biopsicosocial y de género.

Descriptores: Trastornos Psicosomáticos; Género; Disparidades en el Estado de Salud; Modelos Biopsicosociales.
\end{abstract}

\begin{abstract}
Objective: to describe psychosocial and cultural factors that can determine the psychosomatic process, to analyze the influence of gender on their development and reflect on how to approach them. Method: this narrative review gathered substantiated content on psychosomatization and discomforts without apparent organic cause that take account of gender as a study variable or offer theoretical foundations. The Dialnet, Scielo, Google Academic, and CUIDEN databases were consulted, as well as books, documents, and statistical reports of interest. Results: 10 articles available on the web, 5 print articles, 8 books and monographs, and 3 statistical reports were selected. Conclusion: what is reflected in the literature is that gender inequalities can condition the development of psychosomatic disorders in relation with psychosocial factors. This is expressed as a conspicuous differential morbidity. That given, it is necessary to highlight the importance of holistic health care from a biopsychosocial and gender perspective.
\end{abstract}

Descriptors: Psychosomatic disorders; Gender; Health Status Disparities; Models, Biopsychosocial.

\section{RESUMO}

Objetivo: descrever os fatores psicossociais e culturais que podem determinar o processo psicossomático, analisar a influência que tem o gênero sobre seu desenvolvimento e refletir sobre sua abordagem. Método: foi realizada uma revisão narrativa, reunindo conteúdos fundamentados em psicossomatização e desconfortos sem causa orgânica aparente que levem em consideração o gênero ou forneçam fundamentação teórica. Foram consultadas as bases de dados Dialnet, Scielo, Google Academic e CUIDEN, bem como livros, documentos e análises estatísticas de interesse. Resultados: Foram selecionados no total dez artigos disponíveis online, cinco artigos em papel, oito livros e monografias e três relatórios estatísticos. Conclusão: a literatura reflete que as desigualdades de gênero podem condicionar o desenvolvimento de transtornos psicossomáticos em relação aos fatores psicossociais. Isso se traduz por uma morbidade diferencial notória. Diante disso, é necessário destacar a importância da atenção holística à saúde, a partir de uma perspectiva biopsicossocial e de gênero.

Descritores: Transtornos Psicossomáticos; Gênero; Desigualdade em Saúde; Modelos Biopsicossociais.

\section{INTRODUCCIÓN}

La psicosomatización podría definirse como el proceso mediante el cual las emociones se expresan a través del cuerpo, dando lugar a patologías sin causa orgánica aparente, en cuyo desarrollo juegan un papel fundamental los factores psicosociales que envuelven a la persona y posibles sucesos vitales estresantes a los que se haya visto sometida ${ }^{1,2}$.

En la actualidad, ya forma parte del conocimiento colectivo que cualquier fuente de estrés supone una pequeña mella en nuestro estado de salud general y éstas se van acumulando de forma silente minando nuestra salud. Del mismo modo ocurre con las emociones reprimidas que no se expresan o asimilan adecuadamente ${ }^{3}$.

Autora correspondente: Silvia Reverté-Villarroya. E-mail: silvia.reverte@urv.cat

Editora Científica: Cristiane Helena Gallasch; Editor Associado: Antonio Marcos Tosoli Gomes 
En 1998, las investigaciones sobre el mecanismo de los receptores opioides reforzaron la consolidación del fenómeno psicosomático como causal de ciertos problemas de salud ${ }^{4}$. Estos estudios concluyen que la activación o inhibición de la producción proteica de las células puede verse condicionada por la activación energética generada por los pensamientos ${ }^{3}$, y que aquello que denominamos "mente" - que teóricamente genera dichos pensamientos - se halla repartida a lo largo y ancho de la anatomía humana en forma de receptores señal a los que se denomina receptores de opiáceos $^{3,4}$.

No obstante, el escepticismo por parte de la comunidad biomédica, el desconocimiento del fenómeno y el equívoco constante con relación a su terminología, que no acaba de quedar definida hasta hace relativamente poco ${ }^{1,5}$, da lugar a un importante infradiagnóstico. Ello repercute, de forma paralela, al consiguiente aumento en el diagnóstico de enfermedades tales como aquellas de etiología ansiosa o como trastornos depresivos que, obviamente, acaban siendo sobremedicalizados ${ }^{6}$, y de forma más acusada en mujeres, sumando la biopolítica que atesora el sesgo de género en la asistencia sanitaria ${ }^{7,8}$

Teniendo en cuenta el contexto contemporáneo regido por un sistema patriarcal que modela de forma inherente los factores psicosociales y culturales, este fenómeno queda reflejado en las desigualdades de incidencia, prevalencia y desarrollo de determinadas patologías en función de género ${ }^{8,9}$.

Las presiones y limitaciones sociales, los ideales de belleza, la imposición del rol, las cargas familiares o el techo de cristal son solo algunos de los fenómenos que han ido -y continúan- mermando el cuerpo y mente de las mujeres, pudiendo ser los desencadenantes de manifestaciones de origen psicosomático ${ }^{10,11}$.

Aparecen entonces los problemas de salud sin causa orgánica aparente, como las migrañas nocturnas, el insomnio, la depresión, la fibromialgia o la incapacidad de experimentar placer ${ }^{1,8}$. Según el modelo psicosomático, no son más que la forma que tiene el organismo de expresar un bloqueo emocional; bloqueo propiciado, en el caso que nos ocupa, por una sociedad en que la figura de la mujer se desenvuelve como ente oprimido y desfavorecido en cuestión de determinantes socioculturales.

Ante ello, la solución más habitual es la medicalización del malestar sin reparar en la profundización de la psique ${ }^{4}$, obteniendo una mejoría transitoria que da pie al conformismo momentáneo y al consumo excesivo de psicofármacos que quizá, si se realizara una atención sanitaria holística real, pudieran ser prescindibles ${ }^{8,9,12}$.

Dado el análisis global del estado actual del tema, este artículo tiene como objetivo describir los factores psicosociales y culturales que puedan determinar los procesos psicosomáticos, la influencia que el género tiene sobre su desarrollo y reflexionar sobre su abordaje.

\section{MÉTODO}

Se ha realizado una revisión narrativa crítica de la literatura Ad Hoc en diferentes plataformas: documentos, libros y artículos de revista indexados. Para ello, se han seleccionado las siguientes bases: Scielo, Dialnet, CUIDEN y Google Academic, consultadas entre los meses de diciembre de 2017 y mayo de 2018.

Los criterios de inclusión de los artículos acotan las fuentes a aquellas que hayan sido publicadas en los últimos 10 años, en castellano, inglés o portugués.

En cuanto a los criterios de exclusión, se tuvo en cuenta aquellos que no cumplieran los criterios citados anteriormente, a los que no se pudiera acceder al contenido de texto completo y/o que trataran desórdenes somatomorfos contemplados en el DSM-IV y DSM-5 en lugar de trastornos de psicosomatización.

Los descriptores utilizados fueron: "psicosomatización", "psicosomático/a", "biopsicosocial”, "síntomas psicosomáticos", "mujer" y "género". Se hizo la búsqueda tanto en castellano como en inglés y se utilizó el operador booleano "AND" para combinar los descriptores.

Se complementó con búsqueda secundaria a partir de bibliografía de interés de artículos relacionados en formato papel, libros y documentos especializados en la materia, así como informes estadísticos a nivel nacional.

Mediante la estrategia inicial de búsqueda en las bases de datos se obtuvieron: 65 artículos de Dialnet, 204 de Scielo, 10500 de Google Académico y 37 de CUIDEN. Una vez aplicados los criterios de exclusión se seleccionaron un total de 10 artículos disponibles en red (Figura 1), 5 artículos en formato papel, 8 libros y monográficos y 3 informes estadísticos. 


\begin{tabular}{|c|c|c|c|c|}
\hline FUENTE & TIPO DE BÚSQUEDA & TÉRMINOS DE BÚSQUEDA & $\begin{array}{c}\text { № } \\
\text { RESULTADOS }\end{array}$ & $\begin{array}{c}\text { ARTÍ́CULOS } \\
\text { SELECCIONADOS }\end{array}$ \\
\hline \multirow{2}{*}{ Dialnet } & \multirow{2}{*}{$\begin{array}{l}\text { Básica. } \\
\text { Filtro: Artículo de revista. } \\
\text { Filtro: Texto completo } \\
\text { disponible. }\end{array}$} & $\begin{array}{l}\text { Síntomas } \\
\text { Psicosomáticos }\end{array}$ & 51 & 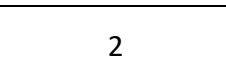 \\
\hline & & $\begin{array}{l}\text { Síntomas } \\
\text { Psicosomáticos AND mujer }\end{array}$ & 14 & 3 \\
\hline \multirow{2}{*}{ Scielo } & \multirow{2}{*}{$\begin{array}{l}\text { Básica. } \\
\text { Acotada a los últimos } 10 \text { años. }\end{array}$} & $\begin{array}{l}\text { Síntomas somáticos AND } \\
\text { mujer }\end{array}$ & 51 & 1 \\
\hline & & $\begin{array}{l}\text { Psicosomático AND } \\
\text { biopsicosocial }\end{array}$ & 153 & 1 \\
\hline $\begin{array}{l}\text { Google } \\
\text { Académico }\end{array}$ & $\begin{array}{l}\text { Básica. } \\
\text { Acotada a los últimos } 10 \text { años. }\end{array}$ & $\begin{array}{l}\text { Biopsicosocial AND mujer } \\
\text { AND psicosomático }\end{array}$ & 10500 & 2 \\
\hline CUIDEN & $\begin{array}{l}\text { Básica. Filtro: Artículos de } \\
\text { revista. }\end{array}$ & Psicosomático & 37 & 1 \\
\hline
\end{tabular}

FIGURA 1: Estrategias de búsqueda en las bases de datos electrónicas. Tortosa, España, 2018.

Fuente: Elaboración propia.

\section{RESULTADOS Y DISCUSIÓN}

\section{Determinantes psicosociales y culturales}

En casi la totalidad de las referencias consultadas se señalan una serie de factores contextuales relacionados con la manifestación de síntomas psicosomáticos, así como la comorbilidad con diferentes patologías sobre todo de carácter psiquiátrico. La literatura respalda que la manifestación de síntomas psicosomáticos sin causa orgánica suele ir asociada en su mayoría de casos a depresión mayor (55\% de los pacientes), trastornos de ansiedad (34\%), de personalidad (61\%) o de pánico (26\%) ${ }^{13}$ así como la mala salud autopercibida, el estrés, el apoyo social o en definitiva, los determinantes de la calidad de vida ${ }^{14,15}$. Asimismo, la diferencia por sexos es más que significativa siendo 10 veces más frecuente la psicosomatización en mujeres que en hombres ${ }^{13}$.

Los estudios publicados revelan la influencia que ejerce el medio sobre el proceso de salud-enfermedad, y en el caso de la mujer, es posible observar cómo significativamente, los condicionantes contextuales de género ejercen como factores de vulnerabilidad. Así, los roles, los estereotipos, los ideales de belleza, la subordinación o el modelo de feminidad son determinantes en el desarrollo de los distintos estilos de vida, enfermedades, el acceso a la sanidad o incluso la mortalidad ${ }^{6,8,16}$.

Partiendo de los determinantes de salud ocupacional, destacan las desigualdades que se producen tanto a nivel salarial como con relación al acceso a puestos de poder, la exclusión de la mujer de estos ámbitos o la dificultad que se presenta con una frecuencia significativamente mayor de conciliación con la vida familiar ${ }^{17,18}$. A destacar que, las profesiones con mayor riesgo psicosocial son aquellas más feminizadas ${ }^{19}$.

Las desigualdades en el mercado laboral tienen una repercusión directa en la salud mental de las mujeres ${ }^{19}$, e implican transversalmente, la imposibilidad de la mujer para "emanciparse" económica y simbólicamente de las directrices que se han estado atesorando en relación con los quehaceres femeninos a lo largo de la historia y romper así con la diferenciación consolidada de aquellas actividades esencialmente femeninas y masculinas ${ }^{20}$. Respecto a ello cabe destacar el fenómeno histórico estructural de dominación sobre la mujer, hecho determinante en la interiorización de inferioridad y sumisión femenina en la sociedad patriarcal occidental ${ }^{18,21}$.

Existen diferencias notables en cuanto a los usos del tiempo en función de género. Las mujeres ocupan más tiempo en realizar tareas domésticas, cuidado de sus hijos/as y familiares dependientes. También otras labores del hogar y estudiar. Por el contrario, el estudio reflejó que, por lo general, los hombres destinaban más tiempo diario al disfrute personal y socializar ${ }^{22}$

El cuidado de las personas dependientes, la crianza de menores y la atención de los mayores son tareas que suelen estar en manos de las mujeres que forman parte del núcleo familiar. Así, para éstas la dedicación a actividades de ocio, deporte, tiempo libre, autocuidado o desarrollo personal se presenta como un gran obstáculo ${ }^{23}$. Todo ello viene reforzado por el sentimiento de no legitimación al empoderamiento y la autodedicación implícita por la ambivalencia que impone la sociedad patriarcal con respecto a la valoración de lo esencialmente femenino ${ }^{18}$.

Paralelamente, cabe resaltar la imposición de los ideales de belleza firmemente instaurado, sobretodo en relación a la figura de la mujer. La presión social inherente, las rebaja a la objetualización y cosificación por parte del mercado publicitario y el ámbito político, constituyendo un potente factor determinante en la autoestima y autoconcepto de las mujeres inmersas en el sistema social de género ${ }^{8,11,21}$. Todo ello queda a su vez reflejado en forma de estadísticas en 
sondeos de la autopercepción de calidad de vida de las mujeres o en la morbilidad diferencial en cuestión de salud mental ${ }^{9,24}$ y manifestaciones de carácter psicosomático ${ }^{16}$.

\section{El malestar de las mujeres}

En la búsqueda del origen del malestar de las mujeres, se realizó una intervención en Atención Primaria a mujeres y hombres con síndromes de malestar (cuadros depresivos o ansiosos, somatizaciones y dolor sin causa orgánica) para hallar los posibles procesos psicosociales asociados. Se registraron los procesos psicosociales de vulnerabilidad distribuidos por sexos, que revelaron que, con un $27 \%$, el principal determinante psicosocial para las mujeres es la carga del rol tradicional (dependencia, aislamiento y falta de red social y el trabajo de cuidadora). A ello le siguen los conflictos de pareja con un $20 \%$ y la preocupación por los hijos. Los duelos, los malos tratos, conflictos en el trabajo/estudios, o la doble jornada laboral son factores con una importancia de peso en los resultados obtenidos ${ }^{16}$.

Para los varones, en cambio, los conflictos de pareja se convierten en el primer factor a recalcar con un $25 \%$ de los casos. Los duelos $(20,3 \%$ y los conflictos laborales o en los estudios $(14,1 \%)$ van seguidos por las cargas del trabajo de cuidador y los accidentes y enfermedades propias (7,8\% en ambos). A la luz de los resultados, se concluye la perpetuidad de los conflictos en función del patrón de los roles de género ${ }^{16}$.

En mujeres amas de casa la probabilidad de depresión aumenta cuando los hijos son autónomos, hecho que sugiere la relación de los estados depresivos con la pérdida del rol social establecido históricamente ${ }^{11}$. Este rol es integrado desde edades muy tempranas mediante la construcción del género e inconscientemente forma la base de la diferencia de los posibles conflictos que pueden desencadenar un proceso de psicosomatización, y del mismo modo, la probabilidad diferencial de desarrollarlo ${ }^{15,25,26}$.

Los malestares culturales experimentados por la mujer influyen directamente en la construcción de la subjetividad individual, pudiendo así generar psicosomatización. Existe una condición consolidada que hace de las mujeres el órgano diana de la sociedad y, apelando al ámbito psíquico de las mismas, se genera una compleja correspondencia entre cuerpo, imagen, normas y estereotipos, haciendo mella poco a poco en el desarrollo vital de las mujeres ${ }^{26}$. Con ello se subraya la importancia no solo del género como construcción social, sino también en la importancia de los procesos hormonales e intrapsíquicos vinculados a la vivencia y simbolización de la diferencia sexual. Esta diferencia inherente hace que ya desde los cimientos la mujer presente una predisposición al desarrollo de enfermedades psicosomáticas, siendo ulteriormente potenciada por los dogmas socioculturales de la sociedad en que se ve envuelta ${ }^{25-27}$.

Se aprecia así la desvalorización de la sociedad patriarcal hacia la categoría femenina frente a la dominancia social masculina, dando lugar de este modo a fuertes inhibiciones conductuales y de pensamiento asociadas a diferentes respuestas emocionales negativas, véase la ansiedad, el miedo o la ira, todas generadoras de malestar personal ${ }^{28,29}$.

\section{La expresión del malestar y su abordaje}

Entre las principales variables socioculturales que constituyen factores protectores se encuentran las redes de apoyo social, el nivel socioeconómico o la cultura de pertenencia. También aquí existen divergencias individuales en función del sexo, sobre todo, en esta última, al tratarse de una sociedad básicamente patriarcal. El impacto de los factores de riesgo puede ser -o no- modulado por estos factores protectores no solamente con anterioridad a la aparición de una enfermedad de origen psicosomático sino también en el desarrollo de esta, pudiendo incluso incidir en el pronóstico ${ }^{30}$.

Estas variables psicológicas que constituyen factores de riesgo se repiten a lo largo de muchos de los documentos consultados. La bibliografía evidencia la influencia inherente de los mensajes impulsados por la sociedad hacia la figura de la mujer, ya sea en cuanto a su cuerpo o apariencia ${ }^{25}$, sus deberes reproductivos ${ }^{27}$, sus limitaciones laborales ${ }^{31} 0$, en definitiva, su subyugación por cuestión de género ${ }^{30}$.

No obstante, no solo la calidad de vida, la ansiedad o el estrés que puedan venir dados de estos fenómenos, se asocian a las correlaciones psicosomáticas en los problemas de salud. Algunas investigaciones apuntan a conflictos emocionales de mucho mayor recorrido. Los estresores tempranos como la separación materna temprana o el maltrato infantil son conflictos que interfieren directamente en factores que influirán en la calidad de vida y la gestión de recursos de afrontamiento y de gestión del estrés en la etapa adulta ${ }^{14}$.

Hombres y mujeres tienden a expresar y canalizar sus malestares de forma diferente. Mientras ellos se inclinan inconscientemente a expresar rabia o refugiarse en hábitos tóxicos, las mujeres tienden a reprimir las emociones generadas por los $\operatorname{conflictos}^{10}$, lo que según la teoría psicosomática generaría una respuesta somática que haría que tuviese lugar una manifestación física sin origen orgánico ${ }^{3,4}$.

Cabe destacar aquellas labores casi exclusivamente femeninas heredadas del sistema patriarcal que no hacen sino contribuir a que tenga lugar este fenómeno. Claro ejemplo es la dificultad de conciliación de la vida laboral y familiar, o sin ir más lejos la ausencia de tiempo libre sin tener que dedicar tiempo a las labores del hogar o al cuidado de las 
personas dependientes ${ }^{11}$. Es por esta doble jornada laboral que en Cataluña las mujeres hacen menos ejercicio físico que los hombres en su tiempo libre, hecho que cuestiona a su vez, la implicación equitativa en educación sanitaria, dado que los discursos de promoción de la salud de las instituciones sanitarias incentivan a los usuarios a aumentar el tiempo de ejercicio diario pero no se repara en la carga que generalmente sustentan las mujeres e impide la realización del mismo, lo que requeriría otro tipo de intervención sanitaria ${ }^{12}$.

Es necesario incidir en el hecho de que el esfuerzo diagnostico inferior hacia las mujeres da lugar a un mayor consumo de ansiolíticos y tranquilizantes ${ }^{11}$, dada la practicidad de recetar un fármaco que calme la sintomatología actual en lugar de indagar en el origen y solventar el problema de base ${ }^{29}$. Ello viene a tener relación con estudios que confirman que la mala salud mental afecta directamente a la salud física, siendo así que personas con patologías de naturaleza ansiosa presentan probabilidades significativamente más elevadas de padecer alguna manifestación somática como cefaleas $(4,2$ veces más riesgo), cardiopatías $(3,9)$, enfermedades osteomusculares $(3,8)$ y enfermedades digestivas ${ }^{10}$.

Hasta un $13 \%$ de las mujeres atendidas en atención primaria salen de la consulta sin un diagnostico propio, con signos y síntomas mal definidos y sin causa médica demostrable ${ }^{16}$. Ante ello, de nuevo surge el fenómeno de la sobremedicalización. A raíz de la escucha clínica insensible y la ausencia de explicación de origen orgánico al malestar de la mujer, se recae en un mayor consumo de tranquilizantes, ansiolíticos e incluso analgésicos ${ }^{26,28,29}$.

Con la intención de erradicar esta omisión clínica del malestar de la mujer, un proyecto de intervención de enfermería en mujeres trabajadoras con perfiles psicosomáticos reflejó la importancia de un abordaje holístico, incidiendo en la labor preventiva de las respuestas estresoras en el ámbito fisiológico, psicológico, sociocultural, del desarrollo y espiritual ${ }^{31}$.

Se destaca el trabajo como un ámbito que proporciona identidad social y debería ser de crecimiento personal para la mujer, siendo de este modo, la prevención de condicionantes negativos una temática de abordaje urgente. Teniendo en cuenta la combinación usual del trabajo productivo y reproductivo casi inherente a la figura de la mujer, implica que los factores psicosociales que la envuelven en el puesto de trabajo pueden ser trasladados al resto de ámbitos y, muchas veces digeridos como malestares sin origen orgánico aparente ${ }^{29,31}$. Es por ello que se designan categorías concretas de intervención centradas en la detección de factores de riesgo y factores protectores, con el fin de establecer las intervenciones necesarias para prevenirlos, contribuyendo de este modo a la calidad de vida de las mujeres trabajadoras ${ }^{31}$.

Estos niveles de prevención e intervención deben hacerse extensibles a la población general, empezando por la desvinculación de la asistencia sanitaria al paradigma biomédico, una concepción más biopsicosocial y con perspectiva de género ${ }^{25,31}$.

\section{CONCLUSIONES}

La literatura consultada evidencia la influencia que ejerce el medio sobre el proceso salud-enfermedad, y cómo los condicionantes psicosociales y culturales de género ejercen como factores de vulnerabilidad. Para las mujeres, los componentes de inferioridad social, es decir, emociones y malestares, derivados de dichos condicionantes, se vuelven factores de riesgo que determinan desde el posible desarrollo de patologías hasta el acceso o atención sanitaria que se le ofrece.

Cabe destacar la desigualdad de género en relación con los factores contextuales, que damnifican a la mujer en cuanto al desarrollo de malestares derivados de la psicosomatización. Se trata de un hecho a tener en cuenta, considerando las presiones y dogmas preestablecidos a los que este porcentaje de la población se ve sometido en la sociedad actual. Por ello, no basta con un enfoque holístico, es necesaria la perspectiva de género para que la asistencia sea del todo integral y no se vea sesgada. La escucha activa y abierta es fundamental para poder detectar hechos y eventos estresantes significativos que puedan actuar como desencadenantes o perpetuadores del malestar de la mujer. Se hace necesario señalar la importancia de la atención sanitaria holística; el modelo de atención ha de basarse en la comprensión de la realidad individual y contextual de cada paciente.

La incidencia y prevalencia de comorbilidades que presentan, en diferentes estudios, como punto de unión, el condicionante de un contexto desfavorable o la perennidad de ciertos patrones ligados a los roles de género sugieren que la educación sanitaria en este aspecto es imprescindible para propiciar un cambio a nivel estructural.

La intervención sanitaria con un enfoque no solo biopsicosocial, sino también de género, estriba de gran importancia. En primer lugar, se hace necesario desmontar la idea de la división mente-cuerpo-emoción. En las manos del personal sanitario radica la responsabilidad de realizar una intervención biopsicosocial competente para con el malestar de las mujeres, teniendo en cuenta que el origen de este malestar puede estribar también en las esferas psicosocial o cultural, no solo en el aspecto biológico.

\section{REFERENCIAS}

1. Marty P. La psicosomática del adulto. París: Amorrortu; 2013 [cited 2017 Dec 03]. 153 p. 
2. Sánchez Medina A. Los trastornos del pensamiento y las enfermedades psicosomáticas. Rev Med [Internet]. 2006 [cited 2017 Dec 19]; 28(4):1346-64. Available from: https://revistamedicina.net/ojsanm/index.php/Medicina/article/view/75-3/447

3. Lipton BH. La biología de la creencia. 5.a ed. Toronto: Palmyra; 2014 [cited 2017 Dec 19]. 286 p.

4. Pert C. Molecules of emotion. New York: Scribner; 2010 [cited 2017 Dec 04]. 368 p.

5. Sánchez-García M. Psychological process in somatization: emotion as process. Rev Int Psicol Ter Psicol [Internet]. 2013 [cited 2018 Jan 12]; 13:255-70. Available from: https://dialnet.unirioja.es/descarga/articulo/4261838.pdf

6. Tortosa Salazar V, Rodríguez Porcel MD, Rodríguez Martínez MM. Trastornos psicosomáticos y ansiedad entre la población: abordaje desde Enfermería. Rev Paraninfo Digit. 2015 [cited 2017 Dec 19]; 22. Available from: http://www.indexf.com/para/n22/pdf/052.pdf

7. Gervás J, Pérez-Fernández M. El encarnizamiento médico con las mujeres. Barcelona: Los libros del lince; 2016 [cited 2018 Jan 20]. $366 \mathrm{p}$.

8. Valls Llobet C. Mujeres Invisibles. 3.a ed. Debolsillo; 2008 [cited 2017 Dec 19]. 384 p.

9. Departament de Salut. Enquesta de Salut de Catalunya (ESCA) 2015: Comportaments relacionats amb la salut, l'estat de salut i I'ús de serveis sanitaris a Catalunya [Internet]. Barcelona: Generalitat de Catalunya; 2016 [cited 2018 Mar 13]. Available from: https://salutweb.gencat.cat/web/.content/_departament/estadistiques-sanitaries/enquestes/Enquesta-de-salut-deCatalunya/Resultats-de-lenquesta-de-salut-de-Catalunya/documents/esca_2015.pdf

10. Velasco Arias S, Ruiz MT, Álvarez-Dardet C. Attention Models to Somatic Symptoms without Organic Cause: from Phisiopathologic Disorders to Malaise of Women. Rev Esp Salud Pública [Internet]. 2006 [cited 2017 Dec 19]; 80(4):317-33. Available from: http://scielo.isciii.es/scielo.php?script=sci_arttext\&pid=S1135-57272006000400003

11. Velasco Arias S. Sexos, género y salud: Teoría y métodos para la práctica clínica y programas de salud. Madrid: Minerva; 2009 [cited 2017 Dec 04]. $352 \mathrm{p}$.

12. Institut Català de les Dones. La salut des de la sensibilitat de gènere. 2.a ed. Barcelona: Generalitat de Catalunya; 2007 [cited 2017 Feb 13]. 58 p.

13. González Ramírez MT, Landero Hernández R. Psychosomatic symptoms and transactional mediational theory of stress. Ansiedad y Estrés [Internet]. 2006 [cited 2018 Apr 29]; 12(1):45-61. Available from: https://dialnet.unirioja.es/servlet/articulo?codigo $=2018974$

14. Berrocal Montiel C, Fava GA, Sonino N. Contributions of Psychosomatic Medicine to Clinical and Preventive Medicine. An Psicol [Internet]. 2016 [cited 2018 Apr 29]; 32(3):828. DOI: https://doi.org/10.6018/analesps.32.3.219801

15. González Ramírez MT, Landero Hernández R. Síntomas psicosomáticos y estrés: comparación de un modelo estructural entre hombres y mujeres. Cienc UANL [Internet]. 2008 [cited 2018 May 25];11(4):403-10. Available from: https://www.redalyc.org/pdf/402/40211412.pdf

16. Velasco Arias S, López Doriga B, Tourné García M, Calderó Bea MD, Barceló Barceló I, Luna Rodríguez C. Evaluation of a biopsycosocial intervention for women ill-being in primary care. Feminismo/s [Internet]. 2007 [cited 2018 May 24]; (10):111-31. DOI: http://dx.doi.org/10.14198/fem.2007.10.08

17. Astelarra J. Veinte años de políticas de igualdad. Madrid: Cátedra; 2005 [cited 2018 May 19]. 400 p.

18. Lasheras Lozano ML, Pires Alcaida M, Rodríguez Gimena MM. Género y Salud - Unidad de Igualdad de Género [Internet]. Sevilla: Instituto Andaluz de la Mujer; 2012 [cited 2018 May 29]. 74 p. Available from: http://www.juntadeandalucia.es/institutodelamujer/ugen/system/files/documentos/97.pdf

19. Vicente-Herrero MT, Ramírez Iñiguez de la Torre MV, Capdevila García L. Salud laboral y género: riesgos laborales. Especial referencia a fertilidad [Internet]. AEEMT; 2018 [cited 2018 May 29]. 34 p. Available from: https://www.uv.es/secretfisio/DOCUMENTO_AEEMT_SALUD_LABORAL_Y_GENERO.pdf

20. Lafaurie Villamil MM. Las mujeres y la depresión: una reflexión crítica. Cuest género la Igual y la Difer [Internet]. 2010 [cited 2018 Apr 29]; (5):315. DOI: http://dx.doi.org/10.18002/cg.v0i5.3791

21. Martínez Verdú R. Psicoanálisis, cultura y sociedad. Cuest género la Igual y la Difer [Internet]. 2007 [cited 2018 May 19 ]; (2):211. DOI: http://dx.doi.org/10.18002/cg.v0i2.3881

22. Matud MP. Usos del tiempo, salud y bienestar de mujeres y hombres. In: Cifre E, Pastor MC, editores. Salud, emociones y género. Castellón de la Plana: Universitat Jaume I; 2015 [cited 2017 Feb 18]. p. 91-105.

23. Pont Martínez P, Santamaría Navarro C, Vicente Herrero MT, Piñaga Solé M, Ramírez Iniguez de la Torre MV, López González AA. Valoración del impacto de género en la salud de mujeres y hombres. Cuest género la Igual y la Difer [Internet]. 2010 [cited 2018 May 29]; (5):51. DOI: http://dx.doi.org/10.18002/cg.v0i5.3780

24. Departament de Salut. Enquesta de Salut de Catalunya (ESCA) 2017: L'estat de salut, elscomportamentsrelacionatsamb la salut, i l'ús de serveissanitaris a Catalunya: resultatsprincipals de l'ESCA 2017 [Internet]. Barcelona: Generalitat de Catalunya; 2017 [cited 2018 Mar 03]. Available from: https://salutweb.gencat.cat/web/.content/_departament/estadistiquessanitaries/enquestes/Enquesta-de-salut-de-Catalunya/Resultats-de-lenquesta-de-salut-de-Catalunya/documents/resumexecutiu_esca_2017.pdf

25. Herrera Salinas D. Aspectos psicosomáticos de la mujer. Rev Fac Cien Med Univ Nac Cordoba [Internet]. 2005 [cited 2018 May 27]; 27:20-31. Available from: http://www.bvs.hn/RFCM/pdf/2005/pdf/RFCMVol2-1-2005-5.pdf

26. Martínez Benlloch I. Patologizar el cuerpo de las mujeres: la contención del malestar. En: Instituto de la Mujer Ministerio de Trabajo e Inmigración, editor. Género y Salud VIII Jornadas de la Red de Mujeres Profesionales de la Salud. Madrid: Debate; 2007 [cited 2018 May 23]. p. 20-31.

27. Pavlova MA. Sex and gender affect the social brain: Beyond simplicity. J Neurosci Res [Internet]. 2017 [cited 2018 Mar 03]; 95 (12):235-50. DOI: https://doi.org/10.1002/jnr.23871 
28. Mingote Adán C. La medicina psicosomática desde una perspectiva de género. In: Mingote Adán C, López-Dóriga Alonso B, editores. II Jornadas de Salud Mental y Género. Madrid: Instituto de la Mujer, Ministerio de Igualdad; 2000 [cited 2018 Feb 23]. p. 15-30.

29. Gismero González E. Una mirada desde el espejo: Consecuencias del abordaje biomédico de las mujeres. Rev Psicoter [Internet]. 2008 [cited 2018 May 27]; 19:69-97. DOI: https://doi.org/10.33898/rdp.v19i76.818

30. Denia Ruiz F, Mingote Adán C. Factores psicosociales que influyen en el desarrollo de la enfermedad coronaria (EC) en la mujer. En: Mingote Adán C, López-Dóriga Alonso B, editores. II Jornadas de Salud Mental y Género. Madrid: Instituto de la Mujer, Ministerio de Igualdad; 2000 [cited 2018 Mar 03]. p. 77-89.

31. Vílchez Barboza V, Paravic Klijn T, Valenzuela Suazo S. Nursing Contribution to Psychophysiologic Disorders of the Working Woman. Enferm Glob [Internet]. 2013 [cited 2018 May 25]; 12(3):254-66. DOI: https://doi.org/10.6018/eglobal.12.3.157191 